(C) 2017 IEEE. Personal use of this material is permitted. Permission from IEEE must be obtained for all other uses, in any current or future media, including reprinting/republishing this material for advertising or promotional purposes, creating new collective works, for resale or redistribution to servers or lists, or reuse of any copyrighted component of this work in other works. 


\title{
A Line Flow Granular Computing Approach for Economic Dispatch with Line Constraints
}

\author{
Xueping Li, Liangxing Fang, Zhigang Lu, Jiangfeng Zhang, Hao Zhao
}

\begin{abstract}
Line flow calculation plays a critically important role to guarantee the stable operation of power system in economic dispatch (ED) problems with line constraints. This paper presents a line flow granular computing approach for power flow calculations to assist the investigation on ED with line constraints, where the hierarchy method is adopted to divide the power network into multiple layers to reduce computational complexity. Each layer contains granules for granular computing, and the layer network is reduced by Ward equivalent retaining the PV nodes and boundary nodes of tie lines to decrease the data dimension. Then Newton-Raphson method is applied further to calculate the power line flow within the layer. This method is tested on IEEE 39-bus and 118-bus systems. The testing results show that the granular computing method can solve the line flow problem in 9.2 $\mathrm{s}$ for the IEEE 118-bus system, while the conventional AC method needs $44.56 \mathrm{~s}$. The maximum relative error of the granular computing method in 300 line flow tests is only $0.43 \%$, which is quite small and acceptable. Therefore, the case studies demonstrate that the proposed granular computing approach is correct, effective, and can ensure the accuracy and efficiency of power line flow calculation.
\end{abstract}

Index Terms-Granular computing, Economic Dispatch, Ward equivalent, Power flow calculation, Hierarchy method.

\section{INTRODUCTION}

$\mathrm{E}$ CONOMIC dispatch (ED) is one of the important measures for the economic operation of power system. Under the premise of ensuring stability of the power system, its aim is to improve socio-economic benefits to the sustainable development of society and economy. ED has attracted extensive attention from the power system community. In the last decades, a number of methods and techniques have been developed and applied to ED, e.g., the gradient method [1], genetic algorithm [2], Tabu search [3], particle swarm optimization [4], and multi-agent technology [5]. These methods and techniques provide feasible solutions to ED.

In general, ED minimizes the generation cost by dealing with generation allocation of generator units, while satisfying power security constraints [6]. In order to maximize the economic benefits, many studies focus on the algorithms to solve the generation allocation problem whilst ignoring the line flow

This work is partially supported by the National Natural Science Foundation of China (No. 61473246), Natural Science Foundation of Hebei Province (No.E2015203294), Qinhuangdao Science and Technology Research and Development Program (201502A010) and China Postdoctoral Science Foundation (No.2014M551049).

XuepingLi, Liangxing Fang, Zhigang Lu, and Hao Zhao are with the Key Lab of Power Electronics for Energy Conservation and Motor Drive of Hebei constraints to simplify the computation, such as [5] and [7]-[9]. The scheduling results which violate secure line constraints will threaten the safety of power system. Hence, economic dispatch with line flow constraints is proposed [10], in which the DC load flow method is applied to calculate the line flows. This method is also adopted in [11]-[13] in similar ED studies. In [14], the concept of generalized generation distribution factor is developed to recalculate line flows when the system generation power is changed, and it is applied to guide practical ED implementations [15]. The generation shift distribution factors are defined in [16]-[17] to obtain new line flows with incremental generator power. The branch power flow sensitivity is also analyzed in [18]-[20]. In the above mentioned methods, nonlinear problems are converted into linear problems, which makes the relevant algorithms perform faster. However, generation allocated in ED by these methods is quite random, and the linearization approach is difficult to handle scenarios where generation power undergoes a significant change. This will decrease the accuracy of the calculation results. Hence, the AC flow methods are used in [21]-[23] to ensure the accuracy of the line flows. Even though, the AC flow calculation for large-scale power systems still needs tremendously long, and sometimes practically infeasible, computing time, and the results could also be non-convergent i.

In order to solve accurately the optimal power flow (OPF) problem for large-scale power systems, the decompositioncoordination principle is developed. A large-scale power system is decomposed into multiple subsystems, where each subsystem is sufficiently small and power flows are calculated in each subsystem independently, then the power flows from all subsystems are coordinated by tie lines to obtain the accurate whole system line flows. To date, several decompositioncoordination algorithms have been studied, such as the Lagrange relaxation algorithm [24], Auxiliary Problem Principle [25], and inner-point method [26]. These methods can also be applied to ED. However, for a large-scale power system, decomposition-coordination algorithms, which need a lot of coordination iterations between subsystems [27] even for small scale systems, has the following issues: If the number of subsystems is small, then the number of nodes in each

Province, Yanshan University, Qinhuangdao, Hebei, 066004, China. (e-mails: lixueping@ysu.edu.cn, 646898403@qq.com, zhglu@ysu.edu.cn).

Jiangfeng Zhang is with the School of Electrical, Mechanical and Mechatronic Systems, University of Technology Sydney, Sydney, Australia (email: jiangfeng.zhang@uts.edu.au) 
subsystem is still very large, and the computation at each subsystem is still very difficult because of large number of variables to be handled; if the number of subsystems is sufficiently big, then the convergence of subsystem coordinations will be difficult to reach.

Overall, existing methods of the power flow calculation in economic dispatch with line constraints for a large-scale power system can be summarized as follows.

1) AC method. It is the most accurate method for calculating the line flows, however, the greater the power system size, the worse the convergence and the computational speed. More seriously, the calculation results might not be convergent for large-scale systems.

2) DC method. It is a more efficient method in terms of computing time, however, it may suffer from low computational accuracy due to the inaccuracy caused by its linearization approach.

3) Decomposition-coordination method. For a large-scale power system, an appropriate balance between the number and size of subsystems is difficult to be found to ensure both the convergence and computing speed.

Granular computing is a kind of methodology that decomposes a complex problem into multiple layers of small scale granules so as to decrease the problem complexity [28]. Granular computing has been applied in many fields, such as the formal concept analysis [29]-[30], human-centered information processing [31], Bayesian analysis of time series [32], abdominal organs detection [33], credit scoring modeling [34], and the granular modeling of signal [35]. In [36], granular computing technique is further improved by the quotient space hierarchy theory from [37]. This hierarchy method can reduce computational complexity effectively, as demonstrated by its successful applications in complex network analysis [38] and the click-stream data warehouse in web servers [39].

Based on the above mentioned hierarchy method, this paper presents a line flow granular computing approach for economic dispatch with line constraints. According to the hierarchy method, the power system is divided into multiple layers. From the top layer to the lowest layer, granules are refined gradually. Coarse granules compute the flows of tie lines, and the computed results will be sent to the lower layer. The finest granules compute the power flows for non-tie lines. Except for the finest granules, equivalent networks for all granules are reduced by Ward equivalent transformation to retain the PV nodes and boundary nodes of tie lines. The presented approach is tested on the standard IEEE 39-bus and 118-bus systems. Simulation results demonstrate that the approach can effectively improve computational speed and data accuracy, and $* * * \% * * * *$ has been $* * * *$.

Compared with existing studies, the main contributions of this work are summarized as follows.

1) Line flow computation process has been significantly accelerated with satisfactory accuracy. This paper applies the hierarchy method to decompose a large-scale power system into multiple layers, where each layer consists of different granules, and each granule has only small number of nodes to avoid curse of dimensionality thus ensure the convergence and computing speed of the power flows calculation. The computation accuracy of the proposed approach is close to conventional AC method, and is much better than that of the DC method.

2) The Ward equivalent transformation retaining the PV nodes and boundary nodes of tie lines is applied to the network to obtain coarse granules. PV nodes retained in the Ward equivalent network provide reactive power support for each PV node and ensure the flows accurately calculated for the tie lines.

3) The application of granular computing in power flow calculation and the Ward equivalent transformation of the top layer to calculate transmission losses are validated in ED which demonstrates the correctness and effectiveness of the proposed approach.

This paper is organized as follows: the model of economic dispatch with line constraints is recalled in Section II. The granular computing approach for economic dispatch with line flow constraints is presented in Section III. A numerical case study is conducted in Section IV. Section V draws conclusions from this work.

\section{Model Of ECONOMIC DisPatch With Line CONSTRAINTS}

\section{A. Objective Function}

The objective of economic dispatch with line constraints is to minimize the total generation cost. It can be formulated as follows:

$$
F_{C}=\sum_{i=1}^{N} \mathbb{d}\left(a \rrbracket_{i} P_{G, i}{ }^{2}+b_{i} P_{G_{i} i}+c_{i}\right)
$$

where $P_{G, i}$ is the power output of generator unit $i ; a_{i}, b_{i}, c_{i}$ are the cost coefficients of the generator unit $i ; N$ is the total number of generator units.

\section{B. Constraints}

1) System power balancing constraints:

$$
\sum_{i=1}^{N} P_{G, i}=P_{D}+P_{\text {Los }}
$$

where $P_{D}$ is the total load demand; $P_{\text {Loss }}$ is the transmission loss.

\section{2) Unit power constraints:}

$$
P_{i}^{\text {min }} \leq P_{G, i} \leq P_{i}^{\max I \frac{1}{1}}
$$

where $P_{i}^{\min P_{i}^{\max } \underline{E}}$ are the minimum and maximum of power output of generator unit $i$, respectively.

\section{3) Line flow constraints:}

$$
-P_{l}^{\max } \leq P_{l} \leq P_{l}^{\max \Pi \mathbb{I}}
$$

where $P_{l}^{\max }$ is the maximum line flow limit of the line $l$.

In the above ED problem with line constraints, the line power flows should be calculated precisely to validate the line flow constraints. Moreover, the computational speed of power flow must be quick enough to meet the need of economic dispatch. 


\section{GRANULAR COMPUTING APPROACH}

\section{A. Hierarchy}

In a problem solving process, not all the detailed system information needs to be considered at the same time. Usually, a complex problem is decomposed and simplified, ignoring some details. Then it is studied from an abstract and simplified level, and then gradually moving into the specific details of the problem. This problem-solving method from a coarse level to a fine one is called as the hierarchy method in [37].

According to the hierarchy method, the power flow calculation problem can be divided into multiple layers to solve. The top layer consists of coarse granules to allow the granular computing approach, from which the granules are gradually refined until all the line flows are calculated at the lowest layer. In order to better clarify the hierarchy method, consider the following power network example in Fig. 1.

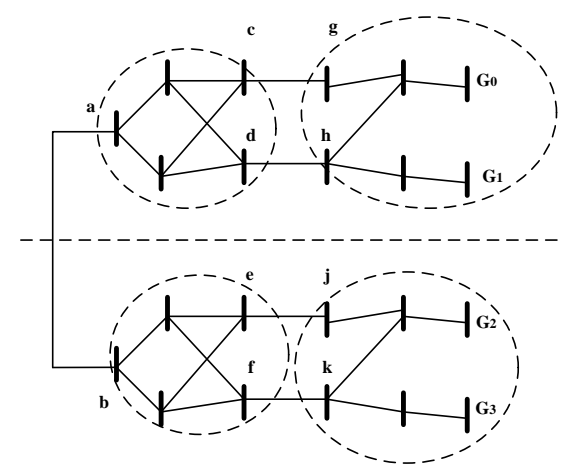

Fig. 1. A power network example

In the network shown in Fig. 1, there are four generation units $G_{0}, G_{1}, G_{\mathbf{2}}$ and $G_{3}$. Assume the network can be divided into four zones, which are indicated by the four circles in Fig. 1. The reason to divide a general power network into small zones is that this zoning technique can effectively reduce power flow computational complexity and speed up the computation, as the number of variables in each small zone is small and the total computational complexity of these zones will be smaller than the original network.. Now each zone in Fig. 1 has one or two tie lines, and the active and reactive power of tie lines become unknown when the power generation is changed following the ED solutions. In the zoning calculation, power flows of tie lines need to be calculated first.

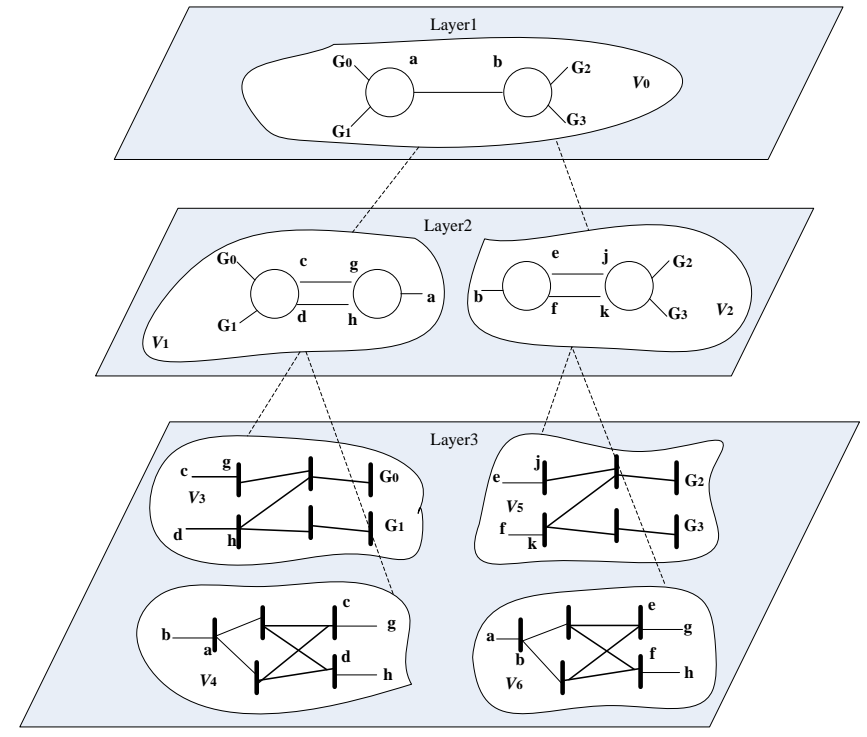

Fig. 2. The three-layered hierarchy.

For the zoning calculation purpose, this network is divided into a three-layered hierarchy as shown in Fig. 2. Each layer has a specific role as described below.

1) Layer 1: This layer has a granule $V_{0}$ to take care the coarse granular computing. Compared to the original network, the network topology of this layer is much simplified, and only four generator nodes, tie-line nodes (the bus $a$ and $b$ ) and related lines are retained. The granular computing method can obtain the power flows on the tie line $a-b$ which will be further allocated to granules of Layer 2.

2) Layer 2: Layer 2 has two granules $V_{\mathbf{1}}$ and $V_{\mathbf{2}}$. The network topology of each granule is also simplified compared to the original network in Fig. 1. This layer can calculate the power flows of tie lines $c-g, d-h, e-j$, and $f-k$, which will be allocated to the granules in Layer 3.

3) Layer 3: Layer 3 has four granules $V_{3}, V_{4}, V_{5}$ and $V_{6}$, which will help to assist the fine granular computing and can determine the power flows of all lines in the original network. It is noted that each of these four granules has also simplified network structure and size compared to the network in Fig. 1.

In the design of these layers, network size and structure should be reduced so as to shorten the computing time, and the corresponding algorithm must also ensure computing accuracy.

\section{B. Network Reduction}

Simplified equivalent network can effectively reduce the number of the network nodes. Ward equivalent transformation is one of these simplification methods to find such an equivalent network, and it has been applied successfully to static security analysis [40], power system planning applications [41], transient stability analysis [42], state estimation [43]. Hence, Ward equivalent network is adopted to reduce complexity in this paper.

Consider the interconnected power system network shown in Fig. 3, where each zone comprises retained nodes (B) and eliminated nodes (E). In a typical Ward equivalent network, retained nodes only include boundary nodes of tie lines, but PV 
nodes should be retained to improve computing accuracy [44]. Hence, retained nodes include PV nodes and boundary nodes of tie lines.

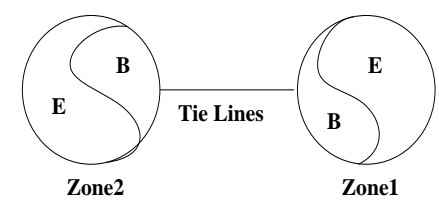

Fig. 3. Interconnected power system network.

Ward equivalent method is applied to each of the above two zones, and the following illustrates how it is applied in a particular zone. Assume the nodal admittance matrix $Y$ of this zone can be expressed as follows [40]:

$Y=$\begin{tabular}{|l|l|}
\hline$Y_{E E}$ & $Y_{E B}$ \\
\hline$Y_{B E}$ & $Y_{B B}$ \\
\hline
\end{tabular}

The nodal admittance matrix $\widetilde{Y}_{B B}$ containing only the retained nodes can be obtained by Gaussian elimination:

$$
\begin{gathered}
\widetilde{Y}_{B B}=Y_{B B}-Y_{B E} Y_{E E}^{-1} Y_{E B} \\
\dot{\tilde{I}}_{B}=\dot{I}_{B}-Y_{B E} Y_{E E}^{-1} \dot{I}_{E}
\end{gathered}
$$

where $\tilde{I}_{B}$ is the equivalent injected current on the retained nodes, which can also be expressed in the form of apparent power and the voltage as follows:

$$
\dot{\tilde{I}}_{B}=\left(\frac{S_{B}}{\dot{U}_{B}}\right)^{*}-Y_{B E} Y_{E E}^{-1}\left(\frac{S_{E}}{\dot{U}_{E}}\right)^{*}
$$

In the power flow calculation, the equivalent power injection is usually used and can be expressed as:

$$
\tilde{S}_{B}=\llbracket \operatorname{diag}\left(\dot{U}_{D_{B}}\right) \tilde{I}_{B}^{*}
$$

In equations (8) and (9), $S_{B}, S_{E}, \dot{U}_{B}$ and $\dot{U}_{E}$ are the power flow data of the base case, which can be obtained by data acquisition.

In Fig. 1, the buses $a$ and $b$ are boundary nodes, $G_{0}, G_{1}, G_{2}$ and $G_{3}$ are generator nodes which are usually set as PV nodes. These nodes are considered as retained nodes. The power network in Fig. 1 can be reduced to obtain granule $V_{0}$ by Ward equivalent method. The reduced network is shown in Fig. 4. The equivalent method of the other layers is the same with that of Layer 1, which is not discussed in details to avoid repetition.

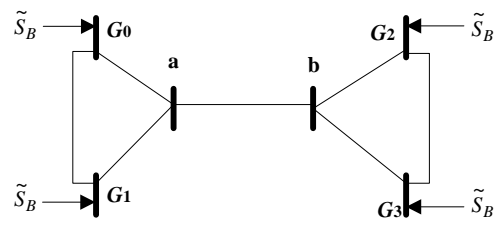

Fig. 4. Reduced network to Layer 1

After Ward equivalent transformation, the nodal admittance matrix $\widetilde{Y}_{B B}$ does not need to be updated. The equivalent power injection $\tilde{S}_{B}$ is updated through the following formula:

$$
\tilde{S}_{B}=\tilde{S}_{B}+\left(S_{B W}-S_{B}\right)
$$

where $S_{B W}$ is the new power injection after the allocation of power generation.

\section{Granular Computing}

In ED, power generation of the units must be allocated before granular computing is implemented,. To satisfy constraints in formulas (2) and (3), transmission loss can be calculated from of the original network or Ward equivalent network.

After the allocation of power generation in ED, the line flow must satisfy constraints in (4), and the line flows will be calculated by the granular computing approach. Flow chart of granular computing is shown in Fig. 5.

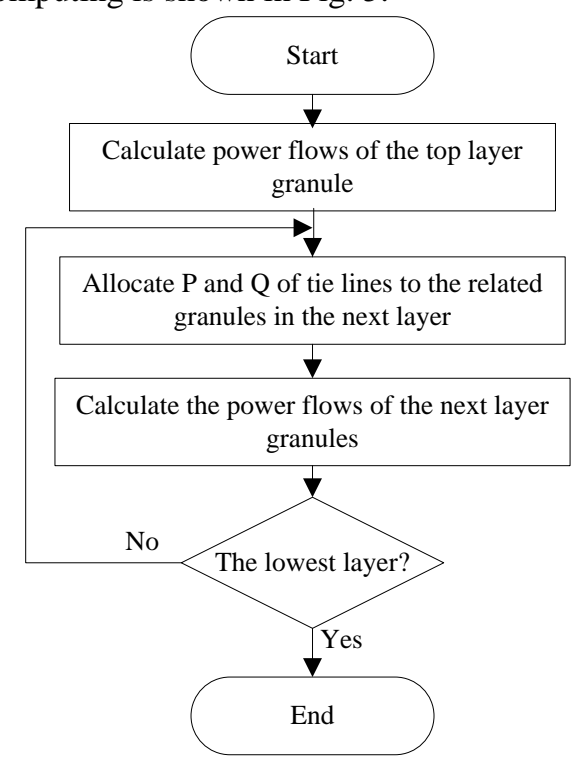

Fig. 5. Flow chart of granular computing

Newton-Raphson method is applied to calculate the AC power flows in each granule. Since the Newton-Raphson method needs a slack bus, at least one PV node will be contained in each granule in order to set the voltage amplitude of the slack bus.

The following steps are implemented in granular computing:

1) Calculate power flows of the top layer granule. In the top granule, the active and reactive power of tie lines can be obtained.

2) Allocate $P$ and $Q$ of tie lines to related granules in the next layer. The active and reactive power of tie lines are allocated to granules of the next layer, which are necessary data to perform power flow calculations in the granules of the next layer.

3) Calculate the power flows for granules in the next layer. With the active and reactive power received from the upper layer tie lines, the power flow of granules in a particular layer can be calculated. If this layer is the lowest layer, then granular computing comes to an end, otherwise it goes back to step 2 .

When granular computing is completed, all the power flows of the original system can be obtained.

For the networks in the granules of a lower layer, the PQ values of the load buses are fixed and given, the values of active 
power at PV buses can be obtained by the results of economic dispatch, while the corresponding values of reactive power $\mathrm{Q}$ are given at PV nodes. Furthermore, the following values still need to be set to calculate the line flows: 1) The boundary nodes of the tie lines: the types of these nodes are set to be PQ nodes, their PQ values come from the upper granules, however, the corresponding node voltage magnitudes and phase angles are unknown, which are set to 1 and 0 respectively; 2) The slack bus: the slack bus is selected as far as possible from PV nodes with large active power, and the selected slack bus should be connected with as many as possible transmission lines. This principle to select slack buses can evenly spread accumulated errors from Ward equivalent reduction to a large number of lines, thus avoid any potential big errors accumulated at a particular line.

\section{Zoning Principle}

Zoning is a key technique to ensure convergence and computational speed of this granular computing method, and it is guided by the following principles:

1) At least one PV node is retained in each zone for the purpose of slack bus selection. Moreover, each zone should not contain too many nodes to ensure convergence.

2) The number of tie lines between different zones needs to be kept as small as possible. Excessive number of tie lines will result in a lot of additional lines after Ward equivalent transformation, which leads to an increase in computing time.

3) The Ward equivalent nodes number of each zone should less than that of its subzones to avoid increase of the computation time.

\section{Simulation Results}

For ED with line constraints, both of the transmission loss and line flow need to be considered. In this paper, line flows are calculated by granular computing, and the transmission losses can be calculated by Ward equivalent network or the original unreduced network. This is because that losses are calculated only after line flows are obtained, and calculation of losses on any of the two network will be quite easy. Here, the transmission losses are calculated by the B-coefficients method on the original network [5], [8], [45].

To assess the effects of granular computing and the Ward equivalent network for ED, two comparison case studies are analyzed, including line flow comparison and ED results comparison.

Case 1 Line Flow Comparison. The purpose of this comparison is to evaluate the line flow calculation accuracy of the proposed granular computing approach. Power generations satisfying line constraints are allocated, in which transmission losses is obtained by B-coefficients method. Based on the power generation results from ED, line flows are calculated by the conventional AC method, DC method, and the newly proposed granular computing approach, respectively. In the conventional AC and DC approaches, the original unreduced network is used. In this comparison study, the AC method is considered to be the most accurate, and thus treated as a benchmark case.

Case 2. ED Result Comparison. The purpose of this comparison is to assess the effect of line flow granular computing method on ED. In this comparison study, three scenarios are tested, which are the ED under AC line flow computation (denoted by ED-AC), ED under DC line flow computation (denoted by ED-DC), and the ED under granular line flow computation (denoted by ED-GC). The original unreduced network is applied for the scenarios ED-AC and EDDC, while the Ward equivalent network is applied for ED-GC. Compared with the ED-AC and ED-DC, the network topology (Ward equivalent network) in ED-GC is the only factor that affects the values of objective function in ED. Hence, the effect of Ward equivalent network on ED is analyzed. Furthermore, to test efficiency of granular computing, the computing time of three dispatch scenarios are compared.

Particle swarm optimization (PSO) [45] is applied to solve the optimization problem in ED. For this PSO, population size is set to 100 , and iteration number is 200 . The tolerance of Newton-Raphson method is set to 0.0001 .

The above comparison studies are conducted both on the IEEE 39-bus system and 118-bus system, where the operation data and parameters are taken from Matpower 5.0 [46]. All programs are coded and tested in MATLAB 7.1 and performed on a PC with an Intel Pentium CPU (3.2GHz) and 8.0GB RAM.

\section{A. IEEE39-Bus System}

In order to evaluate the effect of granular computing, a granular computing case is analyzed. As the IEEE 39-bus system is a small system, in the case, the network is only divided into two layers.

The network is divided into two zones for the power flow calculation. Zoning results are shown in Table I. According to the hierarchy method, we can obtain three granules including a granule in the top layer and two granules in the lower layer. In the top layer, a reduced network is obtained by Ward equivalent method, which is shown in Fig. 6. The network of the top layer is reduced to 16 nodes from 39 nodes, in which the tie lines 25 $26,3-18,14-15$ are retained.

TABLE I

THE ZONES OF IEEE 39-BUS SYSTEM

\begin{tabular}{|c|c|c|}
\hline \multirow{2}{*}{ Zones } & \multicolumn{2}{|c|}{ Retained nodes } \\
\cline { 2 - 3 } & Boundary nodes & $\begin{array}{c}\text { Generator units } \\
\text { nodes }\end{array}$ \\
\hline Zone1 & $15,18,26$ & $33,34,35,36,38$ \\
\hline Zone2 & $3,14,25$ & $30,31,32,37,39$ \\
\hline
\end{tabular}




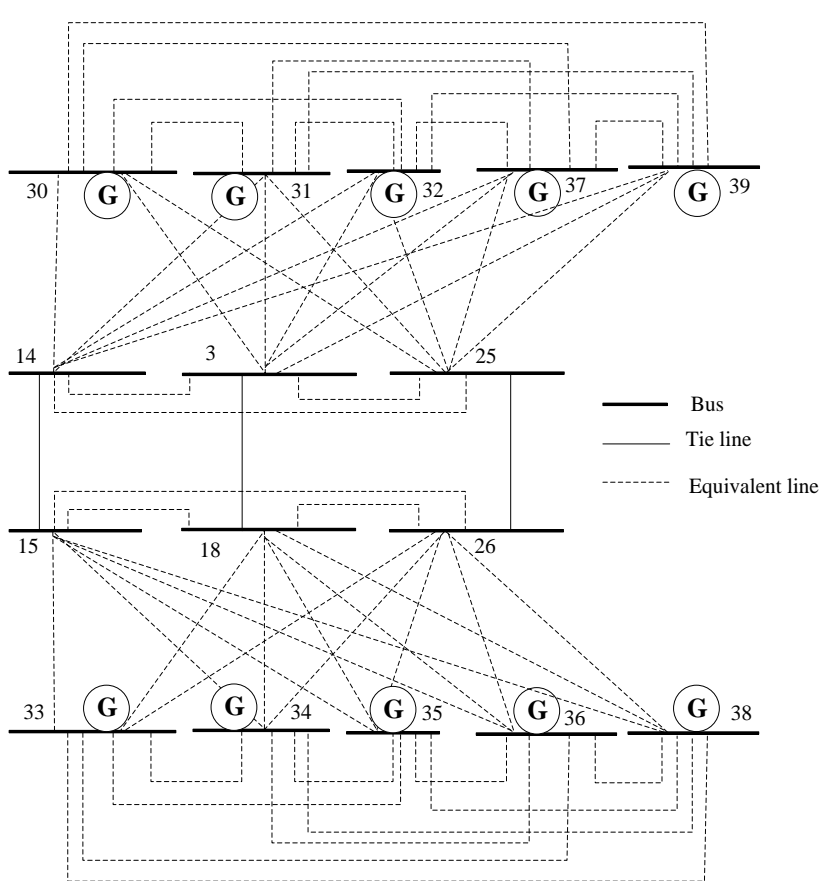

Fig. 6. Reduced network of IEEE 39-bus system to the top layer

After completing the above hierarchy and network equivalent transformation, parameters of 10 generator units are needed for ED study, see Table II for details.

TABLE II

THE PARAMETERS OF IEEE 39-BUS SYSTEM GENERATOR UNITS

\begin{tabular}{|c|c|c|c|c|c|}
\hline Unit & $\begin{array}{r}R_{\mathbb{R}}^{\max } \\
\text { MW }\end{array}$ & $\begin{array}{l}P_{\mathrm{im}}^{\min } \\
\text { MW }\end{array}$ & $\begin{array}{c}a_{\bar{i}} \\
\frac{s}{M W^{2} h}\end{array}$ & $\begin{array}{c}b_{i} \\
s \\
\text { MWh }\end{array}$ & $\begin{array}{l}c_{i} \\
\frac{s}{h} \\
\frac{h}{h}\end{array}$ \\
\hline 30 & 400 & 100 & 0.0199 & 8 & 313 \\
\hline 34,36 & 600 & 200 & 0.0144 & 7 & 390 \\
\hline $31,32,33,35,37$ & 800 & 300 & 0.0092 & 5 & 480 \\
\hline 38 & 1000 & 400 & 0.0082 & 4 & 550 \\
\hline 39 & 1500 & 500 & 0.0073 & 3 & 600 \\
\hline
\end{tabular}

Case 1 Line Flow Comparison: One of the PSO optimization solutions with large generation bias from the base case is selected to analyze the line flow calculation accuracy of granular computing. In the selected solution, power generation is allocated as TABLE III.

TABLE III

THE POWER OF GENERATION UNITS

\begin{tabular}{|c|r|r|}
\hline \multirow{2}{*}{ Unit } & \multicolumn{2}{|c|}{ Power allocated(MW) } \\
\cline { 2 - 3 } & \multicolumn{1}{|c|}{ Base case } & \multicolumn{1}{|c|}{ Selected solution } \\
\hline 30 & 250 & 172.25 \\
\hline 31 & 677.871 & 686.78 \\
\hline 32 & 650 & 737.96 \\
\hline 33 & 632 & 440.37 \\
\hline 34 & 508 & 454.96 \\
\hline 35 & 650 & 777.79 \\
\hline 36 & 560 & 389.35 \\
\hline 37 & 540 & 714.49 \\
\hline 38 & 830 & 901.5 \\
\hline 39 & 1000 & 1020.78 \\
\hline
\end{tabular}

Under the above power injections of the selected solution shown in Table III, the line flows are calculated by the conventional AC method, DC method, and granular computing, respectively. Since only lines with large power flows may exceed line capacity limits, the results of those lines with large power flows are shown in Table IV.

TABLE IV

THE COMPARISON OF FLOW RESULTS

\begin{tabular}{|c|c|c|c|c|c|c|c|c|}
\hline \multicolumn{2}{|c|}{ Line } & \multicolumn{3}{|c|}{ Power flows(MW) } & \multicolumn{2}{|c|}{$\begin{array}{l}\text { Computation } \\
\text { error(MW) }\end{array}$} & \multicolumn{2}{|c|}{$\begin{array}{c}\text { Error in } \\
\text { percentage }(\%)\end{array}$} \\
\hline From & To & $\begin{array}{l}\text { Conventi- } \\
\text { onal AC }\end{array}$ & DC & $\begin{array}{c}\text { Granular } \\
\text { computing }\end{array}$ & DC & \begin{tabular}{|c|} 
Granular \\
computing
\end{tabular} & DC & $\begin{array}{c}\text { Granular } \\
\text { computing }\end{array}$ \\
\hline 29 & 38 & -895.32 & 901.50 & 95.31 & 6.18 & -0.01 & -0.69 & 0.00 \\
\hline 22 & 35 & -777.79 & 777.79 & -777.79 & 0.00 & 0.00 & 0.00 & 0.00 \\
\hline 10 & 32 & -737.96 & 737.96 & -737.96 & 0.00 & 0.00 & 0.00 & 0.00 \\
\hline 25 & 37 & -711.59 & 4.49 & 11.59 & 2.90 & 0.00 & -0.41 & 0.00 \\
\hline 6 & 31 & -685.59 & & 84.77 & 50.01 & -0.82 & 7.29 & 0.12 \\
\hline 21 & 22 & -597.73 & 2.03 & 8.15 & 4.30 & 0.42 & -0.72 & -0.07 \\
\hline 5 & 6 & -567.09 & .19 & 6.69 & 25.90 & -0.40 & 4.57 & 0.07 \\
\hline 20 & 34 & -452.92 & 454.96 & -452.92 & 2.04 & 0.00 & -0.45 & 0.00 \\
\hline 19 & 33 & -438.95 & 440.37 & -438.94 & 1.42 & -0.01 & -0.32 & 0.00 \\
\hline 23 & 36 & -388.65 & 389.35 & 89.50 & 0.70 & 0.85 & -0.18 & -0.22 \\
\hline 23 & 24 & 18.32 & 317.61 & 318.74 & 0.71 & 0.42 & 0.22 & $\begin{array}{l}-0.13 \\
\end{array}$ \\
\hline 5 & 8 & 329.88 & 326.78 & 9.94 & 3.10 & -0.06 & 0.94 & -0.02 \\
\hline 10 & 11 & .29 & & .64 & 13.96 & -0.35 & -4.08 & -0.10 \\
\hline 26 & 27 & 358.93 & 356.37 & 9.37 & 2.56 & -0.44 & 0.71 & -0.12 \\
\hline 2 & 3 & 386.27 & 408.14 & 5.86 & 21.87 & 0.41 & -5.66 & 0.11 \\
\hline 13 & 14 & 393.42 & 378.70 & .05 & 14.72 & 0.37 & 3.74 & 0.0 \\
\hline 10 & 13 & 395.67 & 381.71 & 5.32 & 13.96 & 0.35 & 3.53 & 0.0 \\
\hline 6 & 7 & 451.94 & 445.11 & 451.89 & \begin{tabular}{|l|}
6.83 \\
\end{tabular} & 0.05 & 1.51 & 0.0 \\
\hline
\end{tabular}

We can see from Table IV that the computation error of granular computing is very small, with the maximum error percentage of $0.22 \%$ (line 23-26), while DC has maximum error percentage of $7.29 \%$.

In order to illustrate further the accuracy of the granular computing method, three hundred line flow error percentages are calculated for lines with large power flows for this IEEE 39-bus system using different ED optimization results, and it is found that the maximum error percentage of granular computing is only $0.29 \%$, see Fig. 7 .

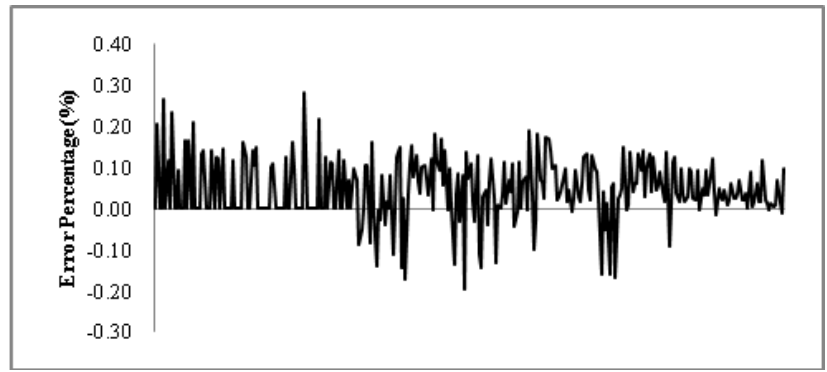

Fig. 7. Three hundred line flow error percentages under granular computing for various ED optimization results

Case 2 ED Result Comparison: Each of the three dispatching scenarios ED-AC, ED-DC and ED-GC is calculated for 50 times. Cost comparison between three dispatch modes for these 
50 trials is shown in Table V. Convergence of the best solution is shown in Fig. 8.

TABLE V

THE COST COMPARISON BETWEEN THREE DISPATCH MODES (50 TRIALS)

\begin{tabular}{|c|c|c|c|}
\hline \multirow{2}{*}{ ED modes } & \multicolumn{3}{|c|}{ Generation cost (\$) } \\
\cline { 2 - 4 } & Max. & Min. & Average \\
\hline ED-AC & 75776.98 & 75776.63 & 75776.75 \\
\hline ED-DC & & & \\
\hline ED-GC & 75785.52 & 75785.22 & 75785.33 \\
\hline
\end{tabular}

From Table $\mathrm{V}$ it is observed that ED-AC and ED-DC have the same generation cost, while ED-GC has a slightly higher generation cost than ED-AC and ED-DC. Actually, it is only $0.01 \%$ higher. In this test, the only factor affecting the ED results of three dispatch modes is the mode of the transmission loss calculation. This also indicates that the difference of the transmission loss calculated by the Ward equivalent network and the original network is small.

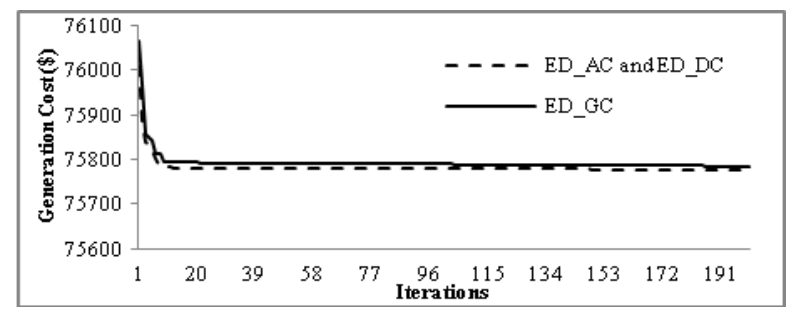

Fig. 8. Convergence of the best solution.

Furthermore, to evaluate computational efficiency, the average computing time of three dispatch modes is shown in Table VI. In this test, the power flow calculation is called when the optimal global solution is updated. From the average power flow computing time in Table VI, it is obvious that ED-DC has the shortest computing time, ED-GC has a shorter computing time than ED-AC.

TABLE VI

THE COMPARISON OF AVERAGE COMPUTING TIME (50 TRIALS)

\begin{tabular}{|c|c|r|}
\hline \multirow{2}{*}{$\begin{array}{c}\text { ED } \\
\text { modes }\end{array}$} & Iterations & Value \\
\hline \multirow{4}{*}{ ED-AC } & Total computing time (s) & 32.478 \\
\cline { 2 - 3 } & Power flow computing time (s) & 2.278 \\
\cline { 2 - 3 } & Number of power flow calculation called & 34 \\
\cline { 2 - 3 } & $\begin{array}{c}\text { Average power flow computing time } \\
\text { in each single call (s) }\end{array}$ & 0.067 \\
\hline ED-DC & Total computing time (s) & 30.506 \\
\hline
\end{tabular}

\begin{tabular}{|c|c|r|}
\hline \multirow{4}{*}{} & Power flow computing time (s) & 0.306 \\
\cline { 2 - 3 } & Number of power flow calculation called & 34 \\
\cline { 2 - 3 } & $\begin{array}{c}\text { Average power flow computing time } \\
\text { in each single call (s) }\end{array}$ & 0.009 \\
\hline \multirow{7}{*}{ ED-GC } & Total computing time (s) & 31.775 \\
\cline { 2 - 3 } & Power flow computing time (s) & 1.575 \\
\cline { 2 - 3 } & Number of power flow calculation called & 35 \\
\cline { 2 - 3 } & $\begin{array}{c}\text { Average power flow computing time } \\
\text { in each single call (s) }\end{array}$ & 0.045 \\
\cline { 2 - 3 } & $\begin{array}{c}\text { Average power flow computing time } \\
\text { of Layer 1 in each single call (s) }\end{array}$ & 0.016 \\
\cline { 2 - 3 } & $\begin{array}{c}\text { Average power flow computing time } \\
\text { of Layer 2 in each single call (s) }\end{array}$ & 0.029 \\
\hline
\end{tabular}

\section{B. IEEE 118-Bus System}

The tested IEEE 118-bus system consists of 54 generation units, in which 19 generator units will be dispatched in ED. The parameters of 19 generator units are shown in Table VII.

TABLE VII

THE PARAMETERS OF IEEE118-BUS SYSTEM GENERATOR UNITS

\begin{tabular}{|c|c|c|c|c|c|}
\hline Unit & $\begin{array}{r}R_{1}^{\max } \\
\text { MW }\end{array}$ & $\begin{array}{c}R_{\mathrm{f}}^{\min } \\
\mathrm{MW}\end{array}$ & $\begin{array}{c}\frac{a_{i}}{s} \\
\frac{s W^{z} h}{}\end{array}$ & $\begin{array}{c}b_{i} \\
\frac{s}{\text { MWh }}\end{array}$ & $\begin{array}{l}c_{i} \\
\frac{s}{h} \\
\frac{h}{h}\end{array}$ \\
\hline 31,87 & 20 & 4 & 0.1561 & 38 & 118 \\
\hline 46 & 76 & 15 & 0.0962 & 14 & 81 \\
\hline $12,54,103,111$ & 100 & 25 & 0.0623 & 18 & 217 \\
\hline $25,49,59,61$ & 350 & 140 & 0.0150 & 11 & 176 \\
\hline $26,65,66,100$ & 400 & 100 & 0.0199 & 8 & 313 \\
\hline 10 & 600 & 200 & 0.0144 & 7 & 390 \\
\hline $69,80,89$ & 800 & 300 & 0.0092 & 5 & 480 \\
\hline
\end{tabular}

The IEEE 118-bus system is a relatively large system and can be used to evaluate the influence of different numbers of granules in granular computing. Based on the system, two scenarios with different zoning divisions are studied. The system zones are shown in Fig. 9 and explained below.

Scenario B.1: The network is divided into two layers, consisting of four granules. Its hierarchy structure is shown in Fig. 10, where the system has three zones: zone 1, 2, and 3 .

Scenario B.2: The network is divided into three layers, consisting of eight granules. Its hierarchy structure is shown in Fig. 11, where zone 1 and zone 2 in Scenario B.1 are further divided into zone 1-1, 1-2, 2-1 and 2-2.

The 19 dispatched generator buses are treated as PV nodes, and the related boundary nodes of tie lines are retained in the reduced networks. 


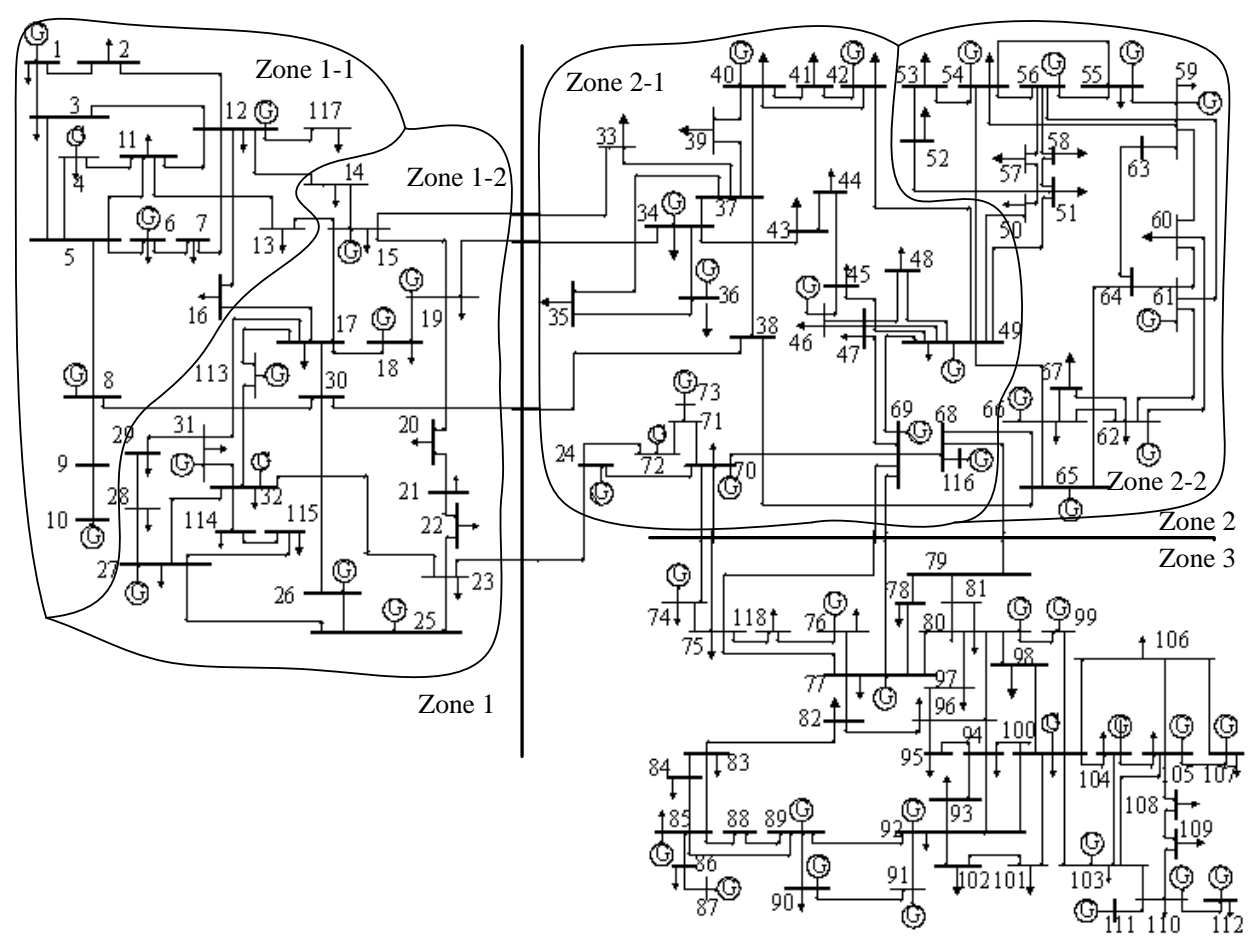

Fig. 9. Zoning division for the IEEE 118-bus system

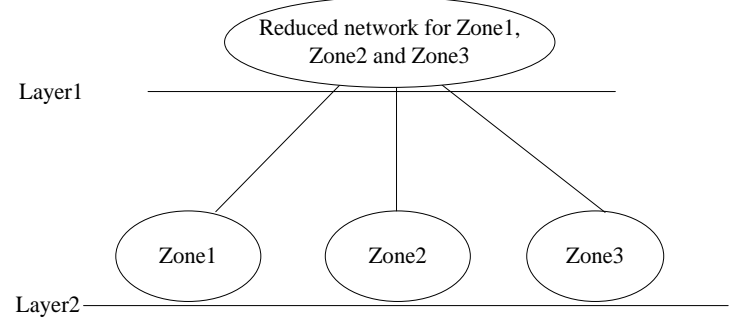

Fig. 10. The hierarchy structure in Scenario B.1

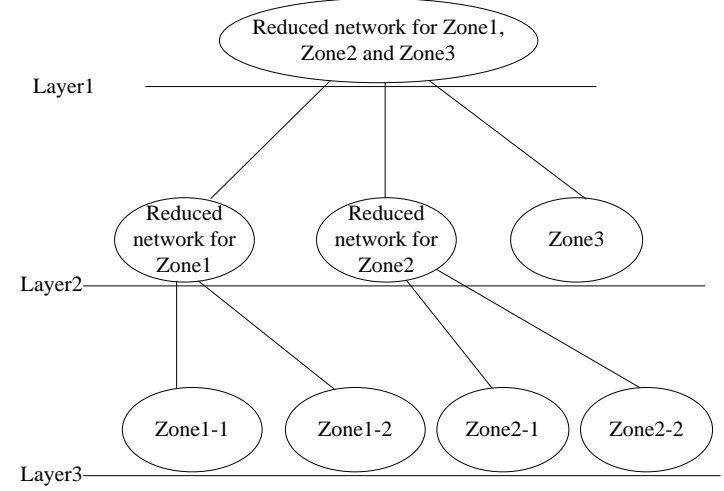

Fig. 11.The hierarchy structure in Scenario B.2
Case 1 Line Flow Comparison: Similar to the analysis of IEEE 39-bus system, one of the solutions with large bias from the base case is selected to analyze granular computing. The comparison of results with large flows is shown in Table VIII.

As seen from Table VIII, the percentage of errors of the DC method is still big (biggest one is 55.94\%), while the worst error percentage of granular computing is only $0.39 \%$ for Scenario B.1 and $0.71 \%$ for Scenario B.2. Moreover, in Scenario B.2, percentage of error is usually small and most of them is below $0.40 \%$. Although the percentage of error for the line $65-68$ reaches $0.71 \%$ in Scenario B.2, its absolute error is only $0.58 \mathrm{MW}$ deviated from the line flow $82.09 \mathrm{MW}$. Therefore, the line 65-68 will not exceed the line flow limit so that it can be ignored in the constraint analysis.

Same as the case study for the 39-bus system, three hundred line flow error percentages are calculated for line flows for Scenarios B. 1 and B. 2 for different ED solutions, and the results are shown in Fig. 12. The maximum percentage of error in Fig. 12 is $0.43 \%$, which does not include the error from the line 6568 as its power is only 82.09 MW which is small and does not exceed the power flow limit. From the analysis above, we consider the two cases are feasible.

TABLE VIII

THE COMPASSION OF FLOW RESULTS

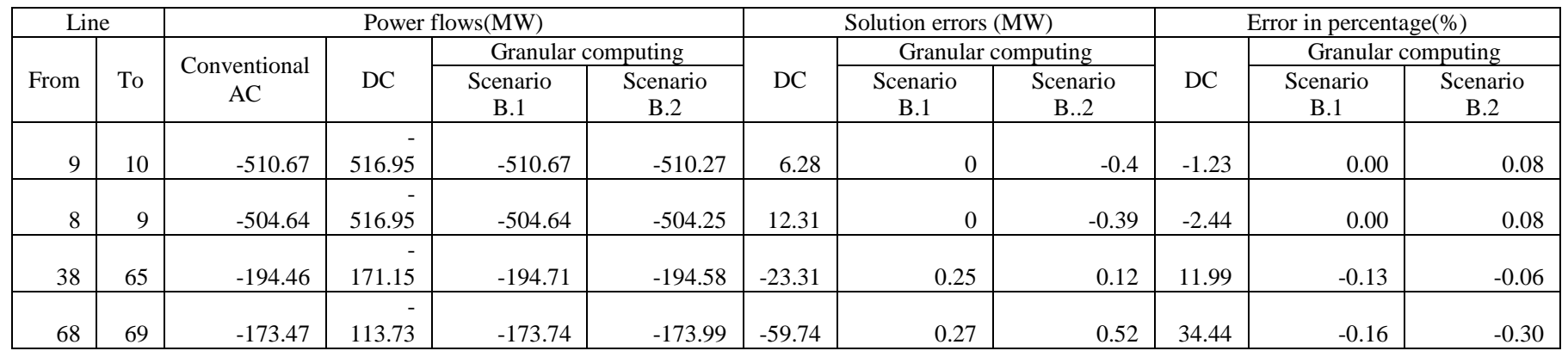




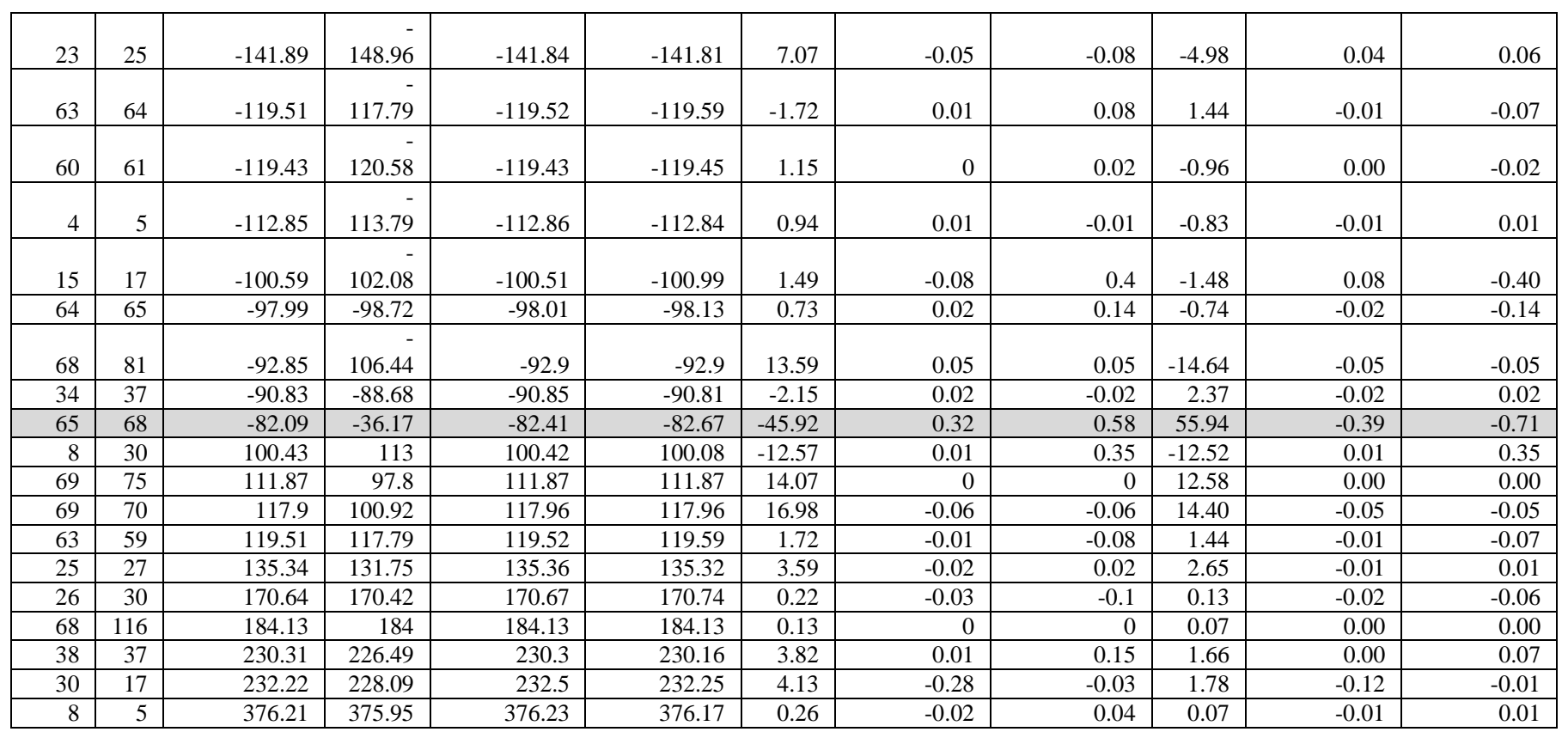

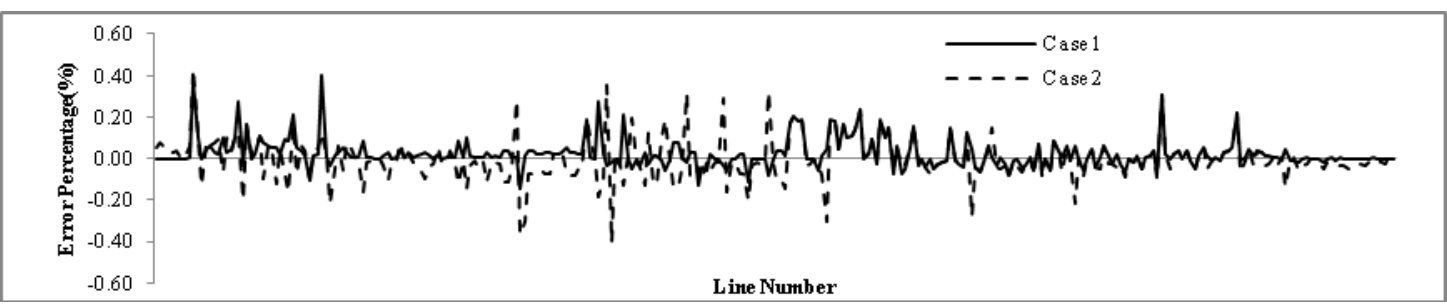

Fig. 12. Three hundred line flow error percentages under granular computing for various ED solutions

Case 2 ED Result Comparison: Through 50 trials, cost comparison between three dispatching modes is shown in Table IX. The percentage of errors for the average generation cost in ED-GC is $0.009 \%$ compared to ED-AC and ED-DC, which is quite small and satisfactory.

TABLE IX

COST COMPARISON BETWEEN THREE DISPATCH MODES (50 TRIALS)

\begin{tabular}{|c|c|c|c|}
\hline \multirow{2}{*}{ ED modes } & \multicolumn{3}{|c|}{ Generation cost (\$) } \\
\cline { 2 - 4 } & Max. & Min. & Average \\
\hline ED-AC & 58911.13 & 58906.24 & 58908.31 \\
\hline ED-DC & & 58910.87 & 58913.68 \\
\hline ED-GC & 58915.35 & 5
\end{tabular}

The average computing time of the three dispatching modes is shown in Table X.

TABLE $X$

THE COMPARISON OF AVERAGE COMPUTING TIME (50 TRIALS)

\begin{tabular}{|c|c|c|c|}
\hline ED modes & & Iterations & Value \\
\hline \multirow{4}{*}{ ED-AC } & \multicolumn{2}{|r|}{ Total computing time $(\mathrm{s})$} & 84.78 \\
\hline & \multicolumn{2}{|c|}{ Power flow computing time (s) } & 44.56 \\
\hline & \multicolumn{2}{|c|}{ Number of power flow calculation called } & 80 \\
\hline & \multicolumn{2}{|c|}{$\begin{array}{l}\text { Average power flow computing time } \\
\text { in each single call (s) }\end{array}$} & 0.557 \\
\hline \multirow{4}{*}{ ED-DC } & \multicolumn{2}{|r|}{ Total computing time (s) } & 41.58 \\
\hline & \multicolumn{2}{|c|}{ Power flow computing time (s) } & 1.36 \\
\hline & \multicolumn{2}{|c|}{ Number of power flow calculation called } & 80 \\
\hline & \multicolumn{2}{|c|}{$\begin{array}{l}\text { Average power flow computing time } \\
\text { in each single call (s) }\end{array}$} & 0.017 \\
\hline \multirow{2}{*}{ ED-GC } & \multirow{2}{*}{$\begin{array}{l}\text { Scenario } \\
\text { B. } 1 \\
\end{array}$} & Total computing time $(\mathrm{s})$ & 50.78 \\
\hline & & Power flow computing time (s) & 10.56 \\
\hline
\end{tabular}

\begin{tabular}{|c|c|c|}
\hline & $\begin{array}{c}\text { Number of power flow calculation } \\
\text { called }\end{array}$ & 80 \\
\hline & $\begin{array}{l}\text { Average power flow computing time } \\
\text { in each single call (s) }\end{array}$ & 0.132 \\
\hline & $\begin{array}{l}\text { Average power flow computing time } \\
\text { of Layer } 1 \text { in each single call (s) }\end{array}$ & 0.049 \\
\hline & $\begin{array}{l}\text { Average power flow computing time } \\
\text { of Layer } 2 \text { in each single call (s) }\end{array}$ & 0.083 \\
\hline \multirow{7}{*}{$\begin{array}{l}\text { Scenario } \\
\text { B.2 }\end{array}$} & Total computing time $(\mathrm{s})$ & 49.42 \\
\hline & Power flow computing time (s) & 9.2 \\
\hline & $\begin{array}{c}\text { Number of power flow calculation } \\
\text { called }\end{array}$ & 80 \\
\hline & $\begin{array}{l}\text { Average power flow computing time } \\
\text { in each single call (s) }\end{array}$ & 0.115 \\
\hline & $\begin{array}{l}\text { Average power flow computing time } \\
\text { of Layer } 1 \text { in each single call (s) }\end{array}$ & 0.049 \\
\hline & $\begin{array}{l}\text { Average power flow computing time } \\
\text { of Layer } 2 \text { in each single call (s) }\end{array}$ & 0.041 \\
\hline & $\begin{array}{c}\text { Average power flow computing time } \\
\text { of Layer } 3 \text { in each single call (s) }\end{array}$ & 0.025 \\
\hline
\end{tabular}

It can be seen from the Table $X$ that the computing time of DC method is the shortest (41.58 s), while the computing time of power flow calculation in the AC method is longest (44.56 s) and accounted for more than $50 \%$ of the total computing time (84.78 s). The power flow computing time of Scenario B.1 and B. 2 in the ED-GC is obviously much smaller than that of the conventional AC. It is noted that the computing time of Scenario B.1 under the ED-GC method is only slightly longer than that of the Scenario B.2. The reasons are as follows: 
The sizes of network Zone 1 and Zone 2 are small, therefore, the time saved for computing the sub-divided network in Layer 2 and 3 of Scenario B.2 is not significant compared to the computing time in Scenario B.1. With the expansion of network scale, granular computing with three layers will have more significant reduced computing time.

\section{Discussions}

Based on the above analysis, the granular computing approach can be applied in ED with line constraints to accelerate the line flow computation process with an acceptable error (maximum relative error is $0.43 \%$ in Fig. 12). Although DC method is the fastest for power flow calculation, its accuracy is poor. The accuracy of the granular computing approach is close to the AC method which is considered to be the most accurate. Under the premise of ensuring computing accuracy, the granular computing approach can significantly accelerate computational speed. From the comparison between the power flow calculation time between the IEEE 39-bus system and the 118-bus system, it is observed that granular computing can significantly reduce computing time for large size power systems. For instance, the AC method needs $44.56 \mathrm{~s}$ to calculate the power flow for the 118-bus system, while the granular computing method needs only $9.2 \mathrm{~s}$. This is mainly because the sizes of the zones in granular computing are small and thus effectively avoid the curse of dimensionality.

\section{CONCLUSION}

This paper aims to solve the accuracy and efficiency problem of line flow computation for ED with line constraints. A line flow granular computing approach for economic dispatch with line constraints is presented, where the hierarchy method is adopted to divide the power network into multiple layers including various granules. For coarse granules of the layers, the network is reduced by Ward equivalent transformation to retain PV nodes and boundary nodes of tie lines and decrease the dimension of variables. Newton-Raphson method is applied in granular computing to calculate the power line flows. The case studies based on IEEE 39-bus and 118-bus systems demonstrate that the proposed granular computing approach is correct and effective, which ensures the accuracy and efficiency of the line flow calculation. For large-scale power network, problems such as non-convergence and slow computing speed can be effectively solved by appropriate hierarchy granular computing.

Acknowledgement The authors are very grateful to the helpful comments from the editors and reviewers which significantly improved the quality of this paper.

\section{REFERENCES}

[1] K. Y. Leel, Y.M. Park, J. L. Ortiz, "Fuel cost minimize at ion for both real and reactive power dispatches," Proc. Inst. Elect. Eng., Gen., Tranm. Distrib., vol.131, no. 3, pp. 85-93, 1984

[2] P. H. Chen and H. C. Chang, "Large-Scale economic dispatch by genetic algorithm," IEEE Trans. Power Syst., vol. 10, no. 4, pp. 1919-1926, Nov. 1995 .
[3] W. M. Lin, F. S. Cheng, and M. T. Tsay, "An improved tabu search for economic dispatch with multiple minima," IEEE Trans. Power Syst., vol. 17, no. 1, pp. 108-112, Feb. 2002.

[4] J. B. Park, Y. W. Jeong, J. R. Shin, and K. Lee, "An improved particle swarm optimization for nonconvex economic dispatch problems," IEEE Trans. Power Syst., vol. 25, no. 1, pp. 156-166, Feb. 2010.

[5] W. T. Elsayed, E. F. El-Saadany, "A fully decentralized approach for solving the economic dispatch problem," IEEE Trans. Power Syst., vol. 30, no. 4, pp. 2179-2189, July, 2015.

[6] B. H. Chowdhury, S. Rahman, "A review of recent advances in economic dispatch," IEEE Trans. Power Syst., vol. 5, no. 4, pp. 1248-1259, Nov. 1990

[7] X. Hao, Y. T. Mou, M. Y. Fu, Z. Y. Lin, "Distributed bisection method for economic power dispatch in smart grid," IEEE Trans. Power Syst., vol. 30, no. 6, pp. 3024-3035, Nov. 2015.

[8] J. P. Zhan, Q. H. Wu, C. X. Guo, etc., "Economic dispatch with nonsmooth objectives-Part I: Local minimum analysis," IEEE Trans. Power Syst., vol. 30, no. 2, pp. 710-721, Mar. 2015.

[9] K. T. Chaturvedi, M. Pandit, and L. Srivastava, "Self-Organizing hierarchical particle swarm optimization for nonconvex economic dispatch," IEEE Trans. Power Syst., vol. 23, no. 3, pp. 1079-1087, Aug. 2008.

[10] R. Podmore, "Economic power dispatch with line security limits," IEEE Trans. Power Appar. Syst., pp. 289-295, Feb. 1974.

[11] R. A. Jabr, "Homogeneous cutting-plane method to solve the securityconstrained economic dispatching problem," Proc. Inst. Elect. Eng., Gen, Transm. Distrib., vol. 149, no. 2, pp. 139-144, Mar. 2002.

[12] R. A. Jabr, A. H. Coonick, and B. J. Cory, "A homogeneous linear programming algorithm for the security constrained economic dispatch problem," IEEE Trans. Power Syst., vol. 15, no. 3, pp. 930-936, Aug. 2000.

[13] T. Yalcinoz, M. J. Short, and B. J. Cory, "Security dispatch using the Hopfield neural network," Proc. Inst. Elect. Eng., Gen., Transm. Dis-trib., vol. 146, no. 5, pp. 465-470, Sep. 1999.

[14] W. Y. Ng, "Generalized generation distribution factors for power system security evaluations," IEEE Trans. Power Appar. Syst., vol.PAS-100, pp. 1001-1005, Mar. 1981.

[15] C. C. Kuo, "A novel coding scheme for practical economic dispatch by modified particle swarm approach," IEEE Trans. Power Syst., vol. 23, no. 4, pp. 1825-1835, 2008.

[16] J. F. Chen, S. D. Chen, "Multi objective power dispatch with line flow constraints using the fast Newton-Raphson method," IEEE Trans. Energy Convers., vol. 12, no. 1, pp. 86-93, Mar. 1997.

[17] J. Y. Fan, L. Zhang, "Real-time economic dispatch with line flow and emission constraints using quadratic programming," IEEE Trans. Power Syst., vol. 13, no. 2, pp. 320-325, May. 1998.

[18] W. R. Barcelo, P. Rastgoufard, "Control area performance improvement by extended security constrained economic dispatch," IEEE Trans. Power Syst., vol. 12, no. 1, pp. 120-128, Feb. 1997.

[19] M. Aganagic, S. Mokhtari, "Security constrained economic dispatch using nonlinear Dantzig-Wolfe decomposition," IEEE Trans. Power Syst., vol. 12, no. 1, pp. 105-112, Feb. 1997.

[20] S. C. Yang, D. Zeng, H. F. Ding, etc., "Stochastic security-constrained economic dispatch for random responsive price-elastic load and wind power," IET. Renew. Power Gener., vol. 10, no. 7, pp. 936-943, July, 2016

[21] N. Amjady, H. N. Rad, "Nonconvex economic dispatch with AC constraints by a new real coded genetic algorithm," IEEE Trans. Power Syst., vol. 24, no. 3, pp. 1489-1502, 2009.

[22] P. K. Hota, A. K. Barisal, R. Chakrabarti, "Economic emission load dispatch through fuzzy based bacterial foraging algorithm," Int. J. Electr. Power Energy Syst., vol. 32, no. 7, pp. 794-803, Sep. 2010.

[23] G. Baskar, M. R. Mohan, "Security constrained economic load dispatch using improved particle swarm optimization suitable for utility system," Int. J. Electr. Power Energy Syst., vol. 30, no. 10, pp. 609-613, Dec. 2008.

[24] A. J. Conejo and J. A. Aguado, "Multi-area coordinated decentralized DC optimal power flow," IEEE Trans. Power Syst.,vol. 13, pp.1272-1278, Nov. 1998.

[25] B. H. Kim, R. Baldick, "Coarse-grained distributed optimal power flow," IEEE Trans. Power Syst., vol. 12, no. 2, pp. 932-939, May. 1997.

[26] W. Yan, L. Wen, W. Li, et al. "Decomposition-coordination interior point method and its application to multi-area optimal reactive power flow," Int. J. Electr. Power Energy Syst., vol. 33, no. 1, pp. 55-60, Jan. 2011.

[27] X. B. Teng, Q. Xia, "A novel decentralized method of multi-area security constraint economic dispatch," DRPT - Int. Conf. Electr. Util. 
Deregulation Restructuring Power Technol., Weihai, Shandong, China, July 9, 2011, pp. 372-377.

[28] Y. Y. Yao, "Perspectives of granular computing," Proc. IEEE Int. Conf. Granul. Comput., Jul. 2005, vol. 1, pp. 85-99.

[29] W. Z. Wu, Y. Leung, J. S. Mi, "Granular computing and knowledge reduction in formal contexts," IEEE Trans. Knowl. Data Eng., vol. 21, no. 10, pp. 1461-1474, Oct. 2009.

[30] W. H. Xu, W. T. Li, "Granular computing approach to two-way learning based on formal concept analysis in fuzzy datasets," IEEE Trans. Cybern., vol. 46, no. 2, pp. 366-379, Feb. 2016.

[31] A. Bargiela, W. Pedrycz, "Toward a theory of granular computing for human-centered information processing," IEEE Trans. Fuzzy Syst., vol. 16, no. 2, pp. 320-330, Apr. 2008.

[32] O. Hryniewicz, K. Kaczmarek, "Bayesian analysis of time series using granular computing approach," Appl. Soft Comput. J., vol. 47, pp. 644652, Jan. 2014.

[33] J. Juszczyk, E. Pietka, B. Pyciński, "Granular computing in model based abdominal organs detection," Comput. Med. Imaging Graph., vol. 46, pp. 121-130, Dec. 2015.

[34] M. Saberi, M. S. Mirtalaie, F. K. Hussain, etc., "A granular computingbased approach to credit scoring modeling," Neurocomputing, vol. 122, pp. 100-115, Dec. 2013.

[35] A. Gacek, "Granular modelling of signals: A framework of granular computing," Inf. Sci., vol. 221, pp. 1-11, Feb. 2013.

[36] J. T. Yao, A. V. Vasilakos, W. Pedrycz, "Granular computing: Perspectives and challenges," IEEE Trans. Cybern., vol. 43, no. 6, pp. 1977-1989, Dec.2 013.

[37] L. Zhang, B. Zhang, "The quotient space theory of problem solving," Fund. Inf., vol. 59, no. 2-3, pp. 287-298, 2004.

[38] L. Zhang, F. G. He, Y. P. Zhang, etc., "A new algorithm for optimal path finding in complex networks based on the quotient space," Fund. Inf., vol. 93, no. 4, pp. 459-469, 2009.

[39] X. H. Chen, Y. J. Wu, H. Y. Cheng, "Quotient space granular computing for the click-stream data warehouse in web servers," CCTAE - Int. Conf. Comput. Commun. Technol. Agric. Eng., vol. 3, pp. 93-96, 2010.

[40] A. Monticelli, S. Deckmann, A. Garcia, etc., "Real-time external equivalents for static security analysis," IEEE Trans. Power Appar. Syst., vol. PAS-98, no. 2, pp. 498-508, Apr. 1979.

[41] E. C. Housos, G. Irisarri, R. M. Porter, etc., "Steady state network equivalents for power system planning applications," IEEE Trans. Power Appar. Syst., vol. PAS-99, no. 6, pp. 2113-2120, Apr. 1980.

[42] T. L. Baldwin, L. Mili, A. G. Phadke, "Dynamic Ward equivalents for transient stability analysis," IEEE Trans. Power Syst., vol. 9, no. 1, pp. 59-67, Feb. 1994.

[43] E. W. S. Ângelos, E. N. Asada, "Improving state estimation with real-time external equivalents," IEEE Trans. Power Syst., vol. 31, no. 2, pp. 12891296, Mar. 2016.

[44] S. Deckmann, A. Pizzolante, A. Monticelli, etc., "Studies on power system load flow equivalencing," IEEE Trans. Power Appar. Syst., vol. PAS-99, no. 6, pp. 2301-2310, Dec. 1980.

[45] Z. L. Gaing, "Particle swarm optimization to solving the economic dispatch considering the generator constraints," IEEE Trans. Power Syst., vol. 18, no. 3, pp. 1187-1195, Aug. 2003.

[46] The Power Systems Engineering Research Center Website, 2016. [Online]. Available: http://www.pserc.cornell.edu/matpower/. 